\title{
Parking functions of types A and B
}

\author{
P. Biane \\ CNRS, Département de Mathématiques et Applications \\ École Normale Supérieure, 45, rue d'Ulm 75005 Paris, FRANCE \\ Philippe.Biane@ens.fr
}

Submitted: May 6, 2001; Accepted: September 30, 2001

MR Subject Classifications: 06A07, 05E25

\begin{abstract}
The lattice of noncrossing partitions can be embedded into the Cayley graph of the symmetric group. This allows us to rederive connections between noncrossing partitions and parking functions. We use an analogous embedding for type B noncrossing partitions in order to answer a question raised by R. Stanley on the edge labeling of the type B non-crossing partitions lattice.
\end{abstract}

\section{Introduction}

A (type $A$ ) parking function is a sequence of positive integers $\left(a_{1}, \ldots, a_{n}\right)$ such that its increasing rearrangement $\left(b_{1}, \ldots, b_{n}\right)$ satisfies $b_{i} \leq i$, while a noncrossing partition of $[1, n]$ is a partition such that there are no $a, b, c, d$ with $a<b<c<d, a$ and $c$ belong to some block of the partition and $c, d$ belong to some other block. The set of noncrossing partitions of $[1, n]$ is denoted by $N C_{n}$, it is a lattice for the refinement order. In $[\mathrm{S}], \mathrm{R}$. Stanley gives a labeling of edges in $N C_{n+1}$, and proves that, through this labeling, parking functions are in one-to-one correspondance with maximal chains in the lattice $N C_{n+1}$.

A type $B$ parking function is a sequence $\left(a_{1}, \ldots, a_{n}\right)$ of positive integers satisfying $a_{i} \leq n$. A noncrossing partition of type $B$, as defined by Reiner $[\mathrm{R}]$, is a noncrossing partition of $\{-1,-2, \ldots,-n, 1,2, \ldots, n\}$ which is invariant under sign change.

In this paper we shall use a natural embedding of $N C_{n+1}$ in the Cayley graph of the symmetric group $S_{n+1}$ to recover Stanley's result. An analogous embedding of $N C_{n}^{B}$ into $W_{n}$, the hyperoctahedral group, then leads to a parallel treatment of the type $\mathrm{B}$ case. In particular we give an edge labeling of $N C_{n}^{B}$ which gives a bijection between maximal chains and type B parking functions, thus answering R. Stanley's question in [S], page 12. The embeddings allow us to use the symmetries of these structures in a very efficient way.

This paper is organized as follows. In the section 2 we describe the embeddings of the non crossing partitions lattices in the corresponding Weyl groups. In section 3 we define 
the edge labelings and show that they yield bijections with the corresponding parking functions.

\section{The embeddings}

Let $G$ be a connected non-oriented graph, with its natural distance. For any pair of vertices $\left(v_{1}, v_{2}\right)$, we call $\left[v_{1}, v_{2}\right]$ the set of all vertices in $G$ which lie on a geodesic (i.e. a path of minimal length) from $v_{1}$ to $v_{2}$. This is an ordered set, in which $v_{1}$ is the smallest element and $v_{2}$ the largest element, while one has $w_{1} \leq w_{2}$ if there exists a geodesic from $w_{1}$ to $v_{2}$ which passes through $w_{2}$, or equivalently there exists a geodesic from $v_{1}$ to $w_{2}$ which passes through $w_{1}$. This ordered set is ranked by the distance from $v_{1}$.

Consider now the Cayley graph built from a Weyl group $W$, taking as generators all the reflexions, and let $w$ be the Coxeter element. We call $N C_{W}$ the ranked ordered set $[e, w]$.

If $W=S_{n}$ is the group of permutations of $[1, n]$, then the reflections are the transpositions, and $w$ is the cycle $(12 \ldots n)$. To any permutation $\sigma \in S_{n}$ we associate the partition of $[1, n]$ given by its cycle structure. This defines a bijection from $N C_{S_{n}}$ to $N C_{n}$, which preserves the order (see e.g. [B1]). In particular an edge $[\tau, \sigma]$ in $N C_{n}$, with $\tau \leq \sigma$, corresponds to a pair of permutations such that $\tau^{-1} \sigma$ is a transposition.

Consider now the case $W=W_{n}$, the hyperoctahedral group. Recall that $W_{n}$ can be identified with the subgroup of $S_{2 n}$, acting on $\{-n,-n+1, \ldots,-1,1,2, \ldots, n\}$, which commutes with the sign change $i \mapsto-i$. The reflections are the transpositions $(i-i)$ and the permutations $(i j)(-i-j)$, with $i \neq j$, which are the even reflexions. The Coxeter element is the cycle $(-1-2 \ldots-n 12 \ldots n)$. The map from $S_{2 n}$ to partitions of $\{-n,-n+1, \ldots,-1,1,2, \ldots, n\}$ defined above restricts to a bijection from $N C_{W_{n}}$ to $N C_{n}^{B}$, see [G], where this is used to recover the type $B$ analogue of the main result in [B2]. Note that the rank function on $N C_{n}^{B}$ does not coincide with the restriction of the rank function on $N C_{2 n}$.

Although we have not looked at this, it would be interesting to investigate the case of other Weyl groups.

\section{$3 \quad$ Labeling of edges}

\subsection{Type A}

As we have seen in the previous section, using the embedding of $N C_{n+1}$ into $S_{n+1}$ every edge $[\tau, \sigma]$ corresponds to a pair of permutations such that $\tau^{-1} \sigma$ is a transposition $(i j)$ where $i<j$. We label such an edge by $i$. This corresponds to the labeling defined by Stanley in [S]. A maximal chain in $N C_{n+1}$ is a sequence of permutations which differ by a transposition, therefore it corresponds to a factorization of $(12 \ldots n+1)$ into a product of $n$ transpositions. 
Theorem 3.1 The map which associates to any factorization

$$
(12 \ldots n n+1)=\left(i_{1} j_{1}\right) \ldots\left(i_{n} j_{n}\right)
$$

into a product of $n$ transpositions, with $i_{k}<j_{k}$, the sequence $\left(i_{1}, \ldots, i_{n}\right)$, is a bijection from the set of all such factorizations to the set of parking functions.

The above considerations show that this is just a rephrasing of Stanley's Theorem 3.1. We shall give a direct proof of this result, since the type B case will be very similar. The map from factorizations to parking functions is straightforward, but given a parking function, finding the associate factorization is not obvious. The proof below gives an algorithm for associating a factorization to any parking function. In particular we do not use the fact that these two sets have the same number of elements. First we remark that there is a natural action of $S_{n}$ on the set of parking functions, which permutes the $a_{j}$. There is also an action of $S_{n}$ on the set of factorizations, which goes as follows. We define an action of the transposition $(k k+1)$ on the set of factorizations. Suppose $(12 \ldots n n+1)=\left(i_{1} j_{1}\right) \ldots\left(i_{n} j_{n}\right)$ is such a factorization, and look at the product $\left(i_{k} j_{k}\right)\left(i_{k+1} j_{k+1}\right)$. There is a unique pair $(u, v)$ with $i_{k}<v ; i_{k+1}<u$ such that $\left(i_{k} j_{k}\right)\left(i_{k+1} j_{k+1}\right)=\left(i_{k+1} u\right)\left(i_{k} v\right)$. We insert this product in the factorization to get a new factorization. One checks that this extends to an action of $S_{n}$ on the set of factorizations. This corresponds to the local action of $S_{n}$ on $V_{N C_{n+1}}$ in [S], Proposition 4.1. Thus we have two actions of $S_{n}$, one on factorizations and one on parking functions, and the map we are looking at is obviously covariant with respect to these actions, therefore in order to prove the theorem it is enough to prove that the restriction of the map to factorizations with nondecreasing $i_{1}, i_{2}, \ldots, i_{n}$ is a bijection with the set of nondecreasing parking functions. We prove this by induction on $n$. We shall make use of the fact

(F) if $\sigma=\sigma_{1} \ldots \sigma_{k}$ is a factorization in $S_{n}$ such that $|\sigma|=\sum\left|\sigma_{i}\right|$ (where $|\sigma|=d(e, \sigma)$ is the length in the Cayley graph) then for each $i$ each cycle of $\sigma_{i}$ is contained in some cycle of $\sigma$ (see e.g. [B1, B2]).

Let $\left(i_{1} j_{1}\right) \ldots\left(i_{n} j_{n}\right)$ be a factorization with $i_{1} \leq \ldots \leq i_{n}$, we claim that $j_{n}=i_{n}+1$. Indeed one has

$$
(12 \ldots n+1)\left(i_{n} j_{n}\right)=\left(12 \ldots i_{n} j_{n}+1 \ldots n+1\right)\left(i_{n}+1 \ldots j_{n}\right)=\left(i_{1} j_{1}\right) \ldots\left(i_{n-1} j_{n-1}\right)
$$

where $i_{1} \leq i_{2} \leq \ldots \leq i_{n-1} \leq i_{n}$ therefore by $(\mathrm{F})$ all transpositions $\left(i_{k} j_{k}\right)$ for $k \leq n-1$ have their support in the set $\left\{1,2, \ldots, i_{n}, j_{n}+1, \ldots, n+1\right\}$, and the cycle $\left(i_{n}+1 \ldots j_{n}\right)$ is the identical permutation. Thus we have

$$
\left(12 \ldots i_{n} i_{n}+2 \ldots n+1\right)=\left(i_{1} j_{1}\right) \ldots\left(i_{n-1} j_{n-1}\right) .
$$

Relabeling $i_{n}+2, \ldots, n+1$ as $i_{n}+1, \ldots, n$, we get a factorization of $(12 \ldots n)$, and since $i_{1} \leq \ldots \leq i_{n-1} \leq i_{n}$, we see by the induction hypothesis that $\left(i_{1}, \ldots, i_{n-1}\right)$ is a parking function of length $n-1$. Since $i_{n} \leq n$, we see that $\left(i_{1}, \ldots, i_{n}\right)$ is a parking function of length $n$. 
Conversely, consider $\left(a_{1}, \ldots, a_{n}\right)$ a nondecreasing parking function. If it comes from some factorization $\left(a_{1} b_{1}\right) \ldots\left(a_{n} b_{n}\right)$, then $b_{n}=a_{n}+1$ as we just saw. But $\left(a_{1}, \ldots, a_{n-1}\right)$ is a non-decreasing parking function of length $n-1$. Since $a_{1}, \ldots, a_{n-1} \leq a_{n}$, relabeling $a_{n}+2, \ldots, n+1$ as $a_{n}+1, \ldots, n$, we see by induction hypothesis that there is a unique factorization

$$
\left(12 \ldots a_{n} a_{n}+2 \ldots n+1\right)=\left(a_{1} b_{1}\right)\left(a_{2} b_{2}\right) \ldots\left(a_{n-1} b_{n-1}\right)
$$

therefore

$$
(12 \ldots n+1)=\left(a_{1} b_{1}\right) \ldots\left(a_{n} a_{n}+1\right)
$$

is the unique factorization corresponding to $\left(a_{1}, \ldots, a_{n}\right)$.

\section{$3.2 \quad$ Type B}

In $N C_{W_{n}}$ the edges are labelled by reflections in $W_{n}$, and the maximal chains thus correspond to factorizations

$$
(-1-2 \ldots-n 12 \ldots n)=r_{1} r_{2} \ldots r_{n}
$$

where $r_{j}$ are reflections.

We shall distinguish three kinds of reflections. For odd reflections i.e. of the kind $(-i i)$ with $i \geq 1$, we label the edge by $i$. For an even reflection of the kind $(i j)(-i-j)$ with $1 \leq i<j$ we label it by $i$, and for an even reflection of the kind $(-i j)(i-j)$ with $1 \leq i<j$, we label it by $j$.

Note that the labels $l(r)$ have the following covariance property with respect to conjugation by the Coxeter element

$$
l\left(w r w^{-1}\right)=c(l(r))
$$

where $c$ is the cyclic permutation $(12 \ldots n)$ acting on $\{1, \ldots, n\}$.

Theorem 3.2 The map which associates, to any factorization

$$
(-1-2 \ldots-n 12 \ldots n)=r_{1} r_{2} \ldots r_{n}
$$

into reflections of $W_{n}$, its sequence of labels $\left(l\left(r_{1}\right), \ldots, l\left(r_{n}\right)\right)$, is a bijection from the set of all factorizations to the set of type $B$ parking functions.

For example the label of the factorization

$$
(-1-2-3123)=[(12)(-1-2)][(3-3)][(-23)(2-3)]
$$

is 133 .

There is again an action of $S_{n}$ on factorizations, similar to the one we had in the type A case, it relies on the fact that any product $r_{1} r_{2}$ of reflections with labels $i_{1}, i_{2}$ can be written uniquely as a product of two reflections $s_{1} s_{2}$ with labels $i_{2}, i_{1}$, as we leave the 
reader to check case by case. Actually we can also make use of the further symmetry (1) which was absent in the type $A$ case. Let $\left(a_{1}, \ldots, a_{n}\right)$ be a type $B$ parking function. Consider all the increasing rearrangements of $\left(c^{k}\left(a_{1}\right), \ldots, c^{k}\left(a_{n}\right)\right)$ for $k=0, \ldots, n-1$, then either these are all equal to $(1,2 \ldots, n)$, or there exists among them some $\left(b_{1}, \ldots, b_{n}\right)$ such that $b_{1}=1$ and $\left(b_{2}, \ldots, b_{n}\right)$ is a nondecreasing parking function. To see this, arrange the $a_{i}$ in increasing order, and consider $m=\max \left\{a_{i}-i \mid 1 \leq i \leq n\right\}$ and $j=\max \left\{i \mid a_{i}-i=m\right\}$. If the $a_{i}$ are not all distinct, then $\left(c^{-j+1}\left(a_{c^{-j+1}(1)}\right), \ldots, c^{-j+1}\left(a_{c^{-j+1}(n)}\right)\right)$ works.

Making use of the actions of $S_{n}$ and of the symmetry (1), it is thus enough to prove the existence of a unique factorization with label $(1,2 \ldots, n)$ or $\left(b_{1}, \ldots, b_{n}\right)$ as above.

The existence is easy. For the first case take

$$
[(1 n)(-1-n)][(2 n)(-2-n)] \ldots[(n-1 n)(-n+1-n)][(n-n)]
$$

For the second, take $r_{1}=(1-1)$ then take the factorization of $(12 \ldots n)$ in $S_{n}$ corresponding to the type $A$ parking function $\left(b_{2}, \ldots, b_{n}\right)$ and symmetrize it to obtain a factorization $r_{2} \ldots r_{n}$ of $(12 \ldots n)(-1-2 \ldots-n)$ with label $\left(b_{2}, \ldots, b_{n}\right)$.

It remains to prove uniqueness of this factorization. We do it in the second case, the first being easy. Let $s_{1} \ldots s_{n}$ be another factorization with the same label. If $s_{1}=(-1,1)$, then by the type $A$ case we are done. If not then $s_{1}=(1 k)(-1-k)$ for some $k$ and

$$
r_{2} r_{3} \ldots r_{n}=(12 \ldots k-1)(-1-2 \ldots-k+1)(k k+1 \ldots n-k \ldots-n)
$$

Since the labels satisfy $b_{2} \leq b_{3} \leq \ldots \leq b_{k} \leq k-1$ it follows from (F) that $r_{2}, \ldots, r_{k}$ have their support in $\{-1, \ldots,-k, 1, \ldots, k\}$ but this is impossible since, the factorization being minimal, $(12 \ldots k-1)(-1-2 \ldots-k+1)$ is the product of at most $k-2$ reflections.

\section{References}

[B1] P. Biane, Some properties of crossings and partitions. Discrete Math. 175 (1997), no. $1-3,41-53$.

[B2] P. Biane, Minimal factorizations of a cycle and central multiplicative functions on the infinite symmetric group. J. Combin. Theory Ser. A 76 (1996), no. 2, 197-212.

[G] F. M. Goodman The infinite hyperoctahedral group and non-crossing partitions of type B. Preprint, 2001.

[M] I. G. Macdonald, Symmetric functions and Hall polynomials, Second Edition, Oxford Univ. Press, Oxford, 1995.

[R] V. Reiner, Non-crossing partitions for classical reflection groups. Discrete Math. 177 (1997), no. 1-3, 195-222.

[S] R. Stanley Parking functions and noncrossing partitions. The Wilf Festschrift (Philadelphia, PA, 1996). Electron. J. Combin. 4 (1997), no. 2. 